\title{
Multiple stress fractures and periorbital hypopigmentation in a young and active adult - a potentially subtle clinical findings of Vitamin D Deficiency
}

Farooq Afzaal ${ }^{1}$, Oran Roche ${ }^{2}$, Preena Patel ${ }^{2}$, and Simon Burtt ${ }^{2}$

${ }^{1}$ Leicester Royal Infirmary

${ }^{2}$ Luton and Dunstable Hospital NHS Foundation Trust

June 15, 2020

\begin{abstract}
21 year old white Caucasian male presented with left foot pain and bilateral periorbital hypopigmentation. An MRI revealed multiple stress fractures. Blood tests revealed Vitamin D deficiency. There is currently evidence confirming relationships between Vitamin D deficiency and cutaneous hypopigmentation. This case suggests further research into this area.
\end{abstract}

Multiple stress fractures and periorbital hypopigmentation in a young and active adult - a potentially subtle clinical findings of Vitamin D Deficiency

${ }^{1}$ Dr Farooq Hassan Afzaal - Luton and Dunstable Hospital

${ }^{2}$ Dr Oran Roche - Luton and Dunstable Hospital (MSK Radiology)Oran.Roche@ldh.nhs.uk

${ }^{3}$ Dr Preena Patel - Luton and Dunstable Hospital (MSK Radiology) Preena.Patel@ldh.nhs.uk

${ }^{4}$ Dr Simon Burtt - Luton and Dunstable Hospital (Trauma and Orthopaedics) Simon.Burtt@ldh.nhs.uk

Multiple stress fractures and periorbital hypopigmentation in a young and active adult - a potentially subtle clinical findings of Vitamin D Deficiency

\section{SUMMARY}


A 21 year old white Caucasian male of Lithuanian origin presented with chronic left foot pain. He presented to the GP and was eventually referred for a specialist orthopaedic opinion. An MRI of his left foot revealed multiple stress fractures and areas of microtrabecular injury. This raised high suspicion for an underlying metabolic bone disorder and the patient was referred to the Rheumatology team for further investigations. Blood tests revealed a severe Vitamin D deficiency $(25 \mathrm{nmol} / \mathrm{L})$ and the patient was investigated for further organic causes. An interesting clinical finding was that of bilateral periorbital hypopigmentation. There is currently little evidence confirming causal relationships between Vitamin D deficiency and cutaneous hypopigmentation. This case prompts further research into this relationship as the earlier detection vitamin D deficiency based on clinical findings could potentiate prompt interventions and therefore minimise the unfavourable effects of an easily treatable clinical condition.

\section{BACKGROUND}

Foot pain is a common presentation in general practice. Whilst a large proportion of presentations result from muskuloskeletal conditions such as plantar fasciitis, some rarer causes of foot pain are a result of bone related disorders such as an accesory navicular, accessory cuboid-os peroneum, bone stress at the base of the fifth metatarsal, bone stress at the base of the second metatarsal and navicular bone stress ${ }^{1}$. Subtle ankle fractures can also present similarly to a sprain and require imaging for further assessment, usually with plain films followed by MRI scans ${ }^{2}$. As the foot is subject to repetitive mechanical forces, it is prone to overuse injuries such as stress fractures, which may be an indication of an underlying pathological process in an otherwise fit and well individual ${ }^{3}$. Stress fractures occur as a result of cyclical injury to bone caused by repeated mechanical stress ${ }^{4}$. Whilst the majority of stress fractures occur in the young and healthy population, particularly in young athletes, there is evidence to suggest that underlying metabolic abnormalities may be responsible for stress fractures in particular cohorts of patients. In particular, patients with reduced bone mineral density due to low dietary intake of calcium or low levels of active vitamin D have a higher risk of developing stress fractures. In women, menstrual disturbances as well as low body index have been identified as risk factors ${ }^{5}$. As foot pain is generally a common presentation, adequate detection of treatable underlying pathological processes is essential for optimum management. Early detection reduces the risk of complications such as intractable pain or non-union, the latter of which requires surgical correction. Whilst literature is present on the link between osteomalacia and stress fractures, little is published on periorbital hypopigmentation and its link with low levels of vitamin D. There is however plentiful literature present on the treatment of hypopigmentation in the form of vitiligo with vitamin $D$ analogues and steroids. This case report highlights a potentially rare presenting feature of a common clinical condition and proposes further research into cutaneous hypopigmentation and its association with vitamin $D$ deficiency and osteomalacia-induced stress fractures.

\section{CASE PRESENTATION}


A 21 year old white Caucasian male of Lithuanian origin presented with chronic left foot pain after working 8-hour long shifts in a casino during the winter months. He reported pain during his work that involves long hours of walking and standing. The patient presented to the GP with generalised foot pain, worse at the base of the 5th metatarsal and at the heel which had gradually been worsening over time.

He reported no significant trauma however complained that the pain was worse on activity and alleviated at rest. There were no complaints of inability to weight bear. The patient was initially referred to the musculoskeletal services for physiotherapy. After achieving little benefit from this, he was referred for a specialist orthopaedic opinion. On initial consultation with the orthopaedic team, he complained of generalised foot pain worse on activity pain and swelling was noted in the region of the 4th metatarsal, which was at risk of increased loading due to a relatively prominent 4th metatarsal head. X-Ray of the left foot revealed a stress fracture through the mid-shaft of the 3rd metatarsal and the patient underwent magnetic resonance imaging (MRI) for further assessment which revealed multiple stress fractures and areas of microtrabecular injury. During his follow up appointment with the orthopaedic team weeks later, there was no tenderness or bruising around the dorsal aspect of the foot or the medial sesamoid. The patient had a full range of movement of the ankle and subtalar joint. The patient reported that following a change in his job, the symptoms had improved dramatically and subsided completely without the need for any intervention. An interesting finding on general examination was the patient's extensive bilateral periorbital hypopigmentation (See figure 1). This coupled with his geographic background, occupational risk factors and confirmation of multiple stress fractures on MRI raised a high suspicion for underlying bone metabolism pathology such as osteomalacia and the patient was investigated further for organic causes of pathological fractures.

\section{INVESTIGATIONS}

Initially the patients foot pain was investigated with plain film imaging. An X-Ray of the left foot revealed a stress fracture through the mid-shaft of the 3rd metatarsal with callus formation and bony erosion in the medial head of the 1st metatarsal (see figure 2). These findings were also evident clinically with tender points on examination. For further visualisation, an MRI of his left foot was performed which revealed multiple stress fractures, particularly affecting the third and fourth metatarsal bones with microtrabecular injury at the navicular, cuboid and the base of the fifth metatarsal (see figres 3,4 and 5). Periostal thickeing and fascial oedema were seen along most of the fourth metatarsal as well as at the distal aspect of the third metatarsal which were findings suggestive of stress fractures. These results raised high suspicion for an underlying metabolic bone disorders such as osteomalacia. The patient was investigated further with blood tests, which confirmed a severe Vitamin D deficiency $(25 \mathrm{nmol} / \mathrm{L})$. The patient was treated with Vitamin D supplements and referred to the Rheumatology team for further investigation of other organic causes. 


\section{TREATMENT}

From an orthopaedic point of view, the patient was advised to wear a walker boot and to be non weight bearing. He was also advised to remove the walker boot to exercise his ankle in order to maintain range of movement and prevent a deep vein thrombosis and subsequent pulmonary embolisms. Based on local protocol, as the patient was able remove the walker boot to exercise his ankle, he did not require chemical thromboprophylaxis. In the follow up consultation, the patient reported not using the walker boot due to inconvenience however reported that only weeks after changing to a less physically demanding job, his symptoms had completely resolved. Regarding the osteomalacia, the patient was advised to commence oral Vitamin D supplementation and referred to the Rheumatology team for screening of underlying metabolic abnormalities. The patient's periorbital hypopigmentation remained unchanged.

\section{OUTCOME AND FOLLOW-UP}

The patient was initially followed up by the orthopaedic department and management conservatively and eventually discharged back to the care of the GP and referred to the Rheumatology department for further investigations. Primarly, the patient underwent blood tests to rule out underlying metabolic pathologies. Bloods tests revealed a vitamin D deficiency suggestive of ostemomalacia and the patient was advised that he will be managed further by the Rheumatology team. As per advice from the rheumatology team, the patient required a metabolic screen to rule out underlying pathologies responsible for the occurence of stress fracuters in an otherwise young, fit and well adult. Common conditions such as ceoliac disease were to be investigated and ruled out with anti-tissue transglutaminase and consideration for a duodenal biopsy as the inability to adequately absorb nutrients from the gut including calcium and vitamin D may lead osteomalacia. Further differentials which needed investigation included congenital hypogonadism which required a hormonal profile and hyperthyroidism which require thyroid function tests as both conditions can be an underlying cause of stress fractures in an otherwise well patient. More rare differentials which were to be investigated included HIV and anorexia nervosa, the latter which would require involvement of the psychiatric team. The patient was discharged from orthopaedics and advised to confirm his next outpatient appointment with the rheumatology which unfortunately he failed to attend.

\section{REFERENCES}


1. Baquie P, Fooks L, Pope J, Tymms G. RACGP - The challenge of managing mid-foot pain [Internet]. Racgp.org.au. 2019 [cited 15 September 2019]. Available from:

https://www.racgp.org.au/afp/2015/march/the-challenge-of-managing-mid-foot-pain/ 2. DB J, DH K. Foot fractures frequently misdiagnosed as ankle sprains. [Internet]. Ncbi.nlm.nih.gov. 2002 [cited 15 September 2019]. Available from: https://www.ncbi.nlm.nih.gov/pubmed/12322769 3. H L, MA D. Cuboid Stress Fractures. [Internet]. Ncbi.nlm.nih.gov. 2019 [cited 15 September 2019]. Available from: https://www.ncbi.nlm.nih.gov/pubmed/31194407 4. Welck M, Hayes T, Pastides P, Khan W, B R. Stress fractures of the foot and ankle. [Internet]. Ncbi.nlm.nih.gov. 2017 [cited 15 September 2019]. Available from: https://www.ncbi.nlm.nih.gov/pubmed/26412591 5. Moreira C, JP B. Stress Fractures: Concepts and Therapeutics. [Internet]. Ncbi.nlm.nih.gov. 2017 [cited 15 September 2019]. Available from: https://www.ncbi.nlm.nih.gov/pubmed/27732325 6. JC M, B K, SE S. Stress fractures of the foot and ankle, part 1: biomechanics of bone and principles of imaging and treatment. [Internet]. Ncbi.nlm.nih.gov. 2017 [cited 15 September 2019]. Available from: https://www.ncbi.nlm.nih.gov/pubmed/28374052 7.https://link.springer.com/article/10.1007\%2Fs10140-016-1390-5 8. Bratke G e. [Which typical foot fractures should the radiologist know?] - PubMed - NCBI [Internet]. Ncbi.nlm.nih.gov. 2019 [cited 21 September 2019]. Available from: https://www.ncbi.nlm.nih.gov/pubmed/29450562 9. Pegrum J e. The pathophysiology, diagnosis, and management of foot stress fractures. - PubMed - NCBI [Internet]. Ncbi.nlm.nih.gov. 2019 [cited 21 September 2019]. Available from: https://www.ncbi.nlm.nih.gov/pubmed/25419892 10. Alghamdi K, Kumar A, Moussa N. The role of vitamin D in melanogenesis with an emphasis on vitiligo. Indian J Dermatol Venereol Leprol. 2013 Nov-Dec;79(6):750-8. 11. McCabe MP e. Current concept review: vitamin D and stress fractures. PubMed - NCBI [Internet]. Ncbi.nlm.nih.gov. 2019 [cited 21 September 2019]. Available from: https://www.ncbi.nlm.nih.gov/pubmed/22735329 12. Spira A, Buttriss JL. Vitamin D: An overview of vitamin D status and intake in Europe. Nutr Bull 2014 Dec; 39(4): 322-350.

https://www.ncbi.nlm.nih.gov/pmc/articles/PMC4288313/ 13. Parsad D, Kanwar AJ. Topical vitamin D3 analogues in the treatment of vitiligo. Pigment Cell Melanoma Res 2009;22:487-8

\section{FIGURE LEGENDS}

Figure 1 - Extensive bilateral periorbital hypopigmentation. Figure 2 - Stress fracture through the midshaft of the 3rd metatarsal with evident healing callus formation. Bony erosion noted in the medial head of the 1st metatarsal, immediately adjacent to the 1st metatarsal phalangeal joint. Figure 3 - MRI T1 Signal - Focal area of linear altered signal on T1 in the bone trabeculation of the navicularis in keeping with microtrabecular/stress injury Figure 4 - MRI STIR sequence - Bone marrow oedema throughout the shaft of the fourth metatarsal with periosteal thickening and surrounding fascial oedema. Features are consistent with an evolving stress reaction/fracture. Figure 5 - MRI STIR sequence - Bone marrow oedema at the distal shaft of the third metatarsal with periosteal thickening and surrounding fascial oedema. Features are suggestive of a stress fracture. 


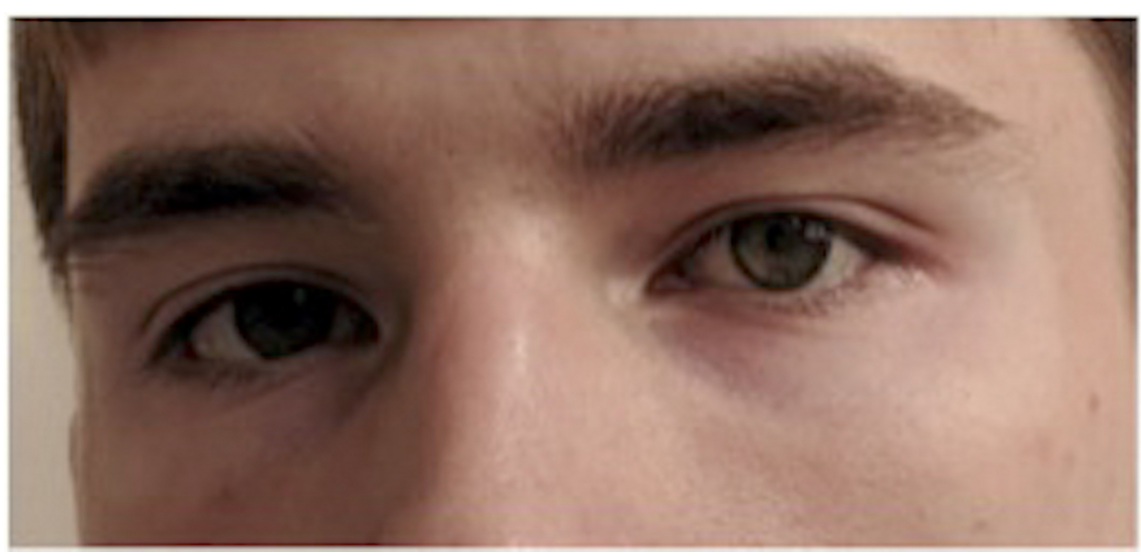




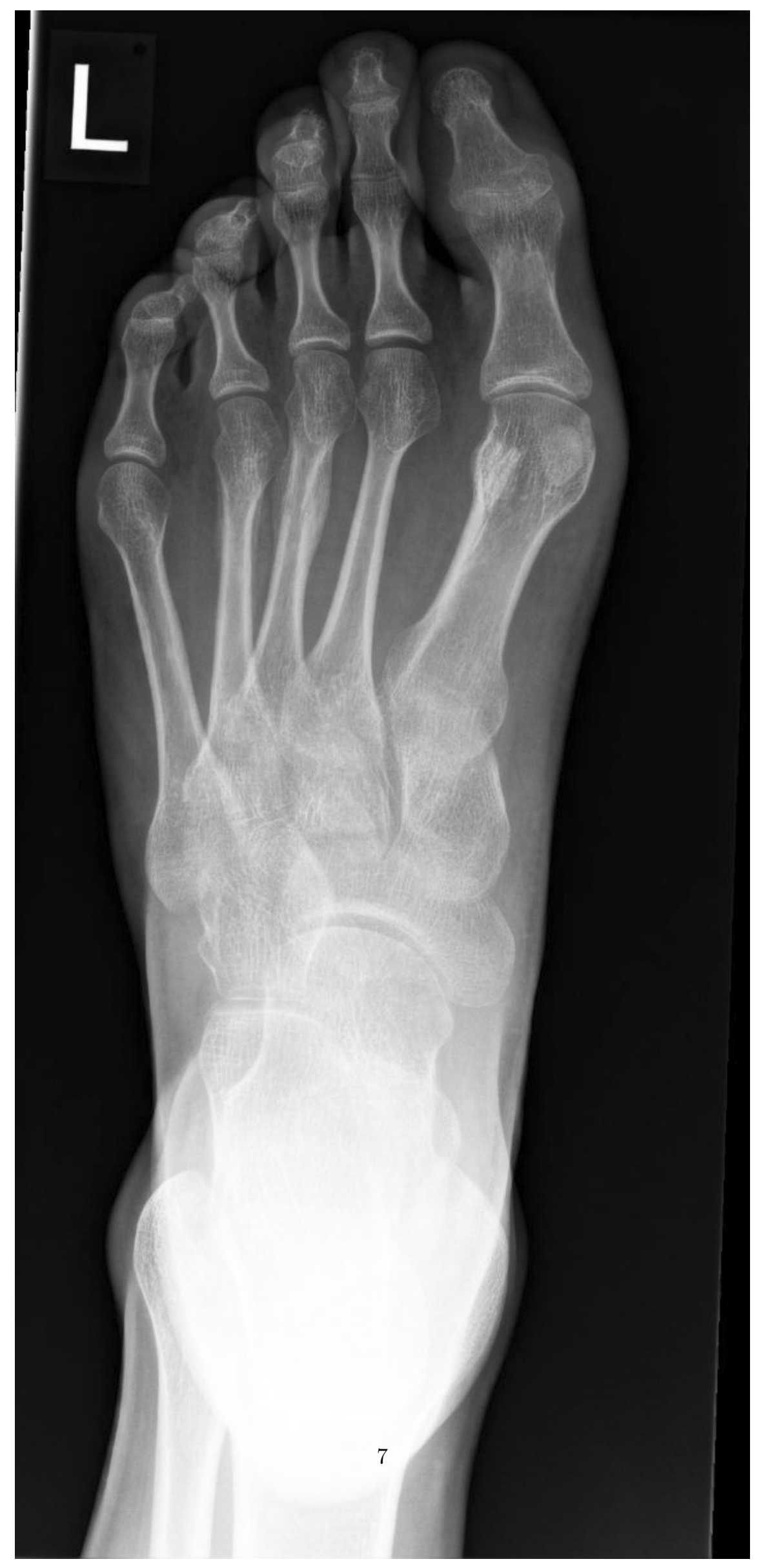




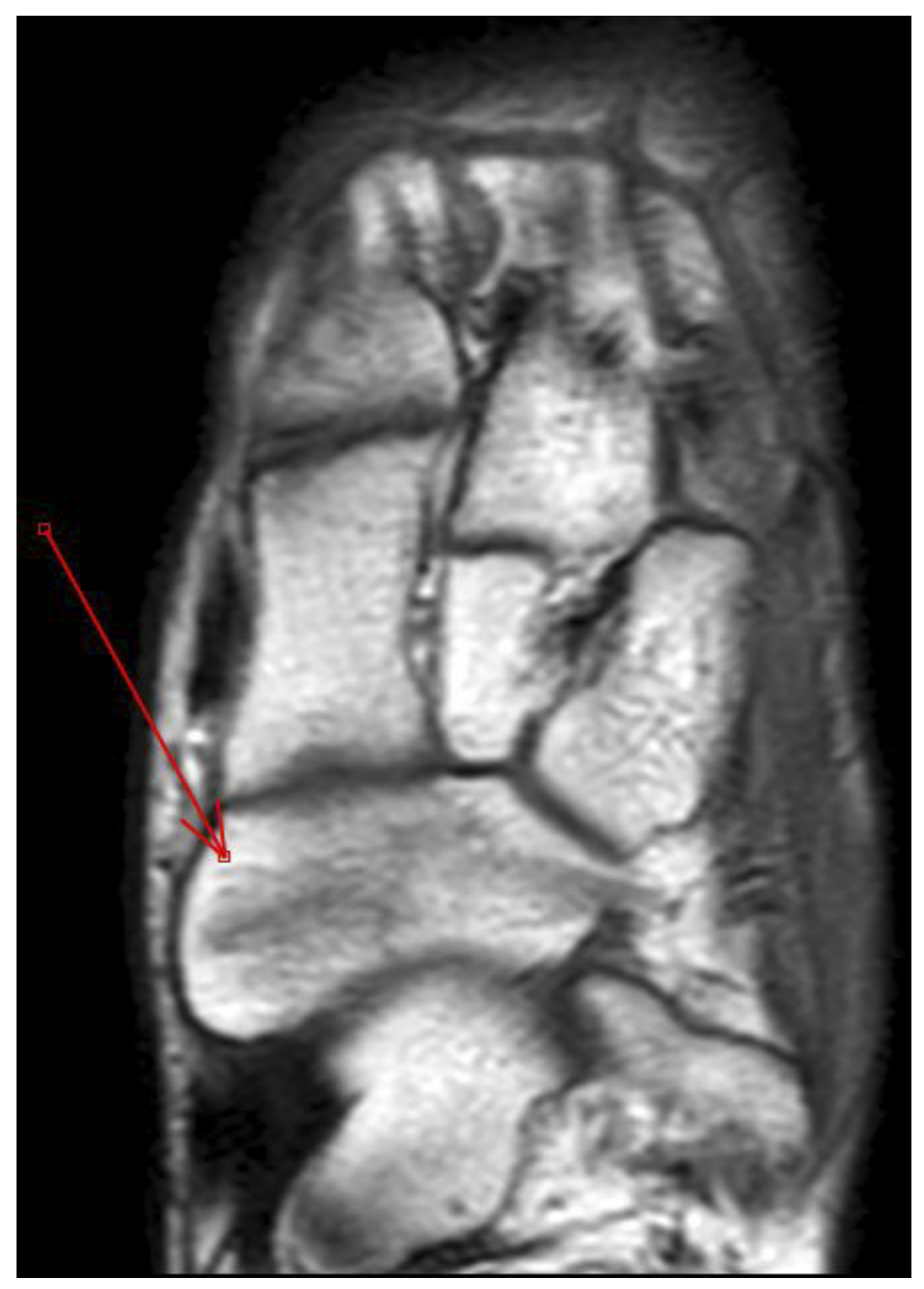




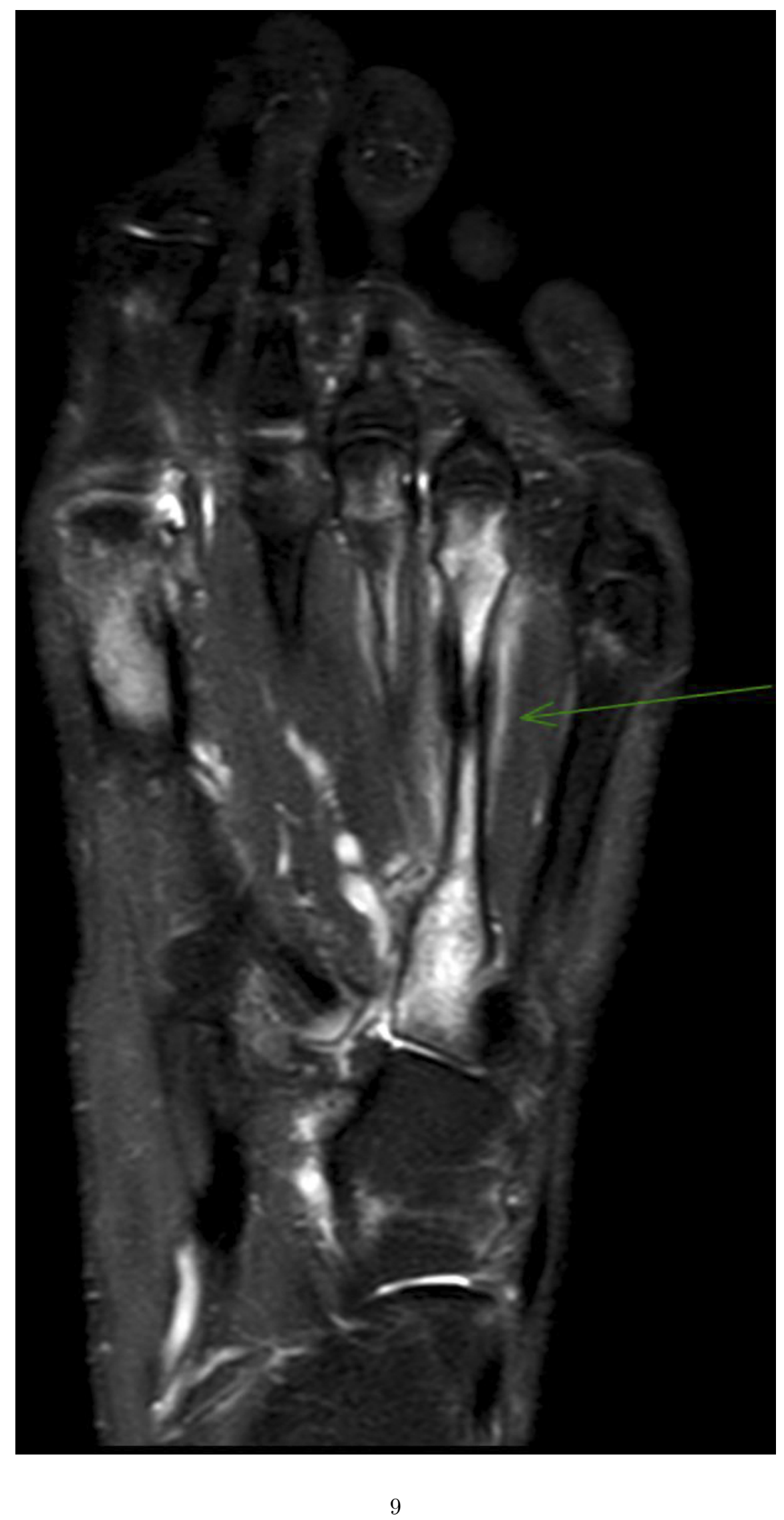




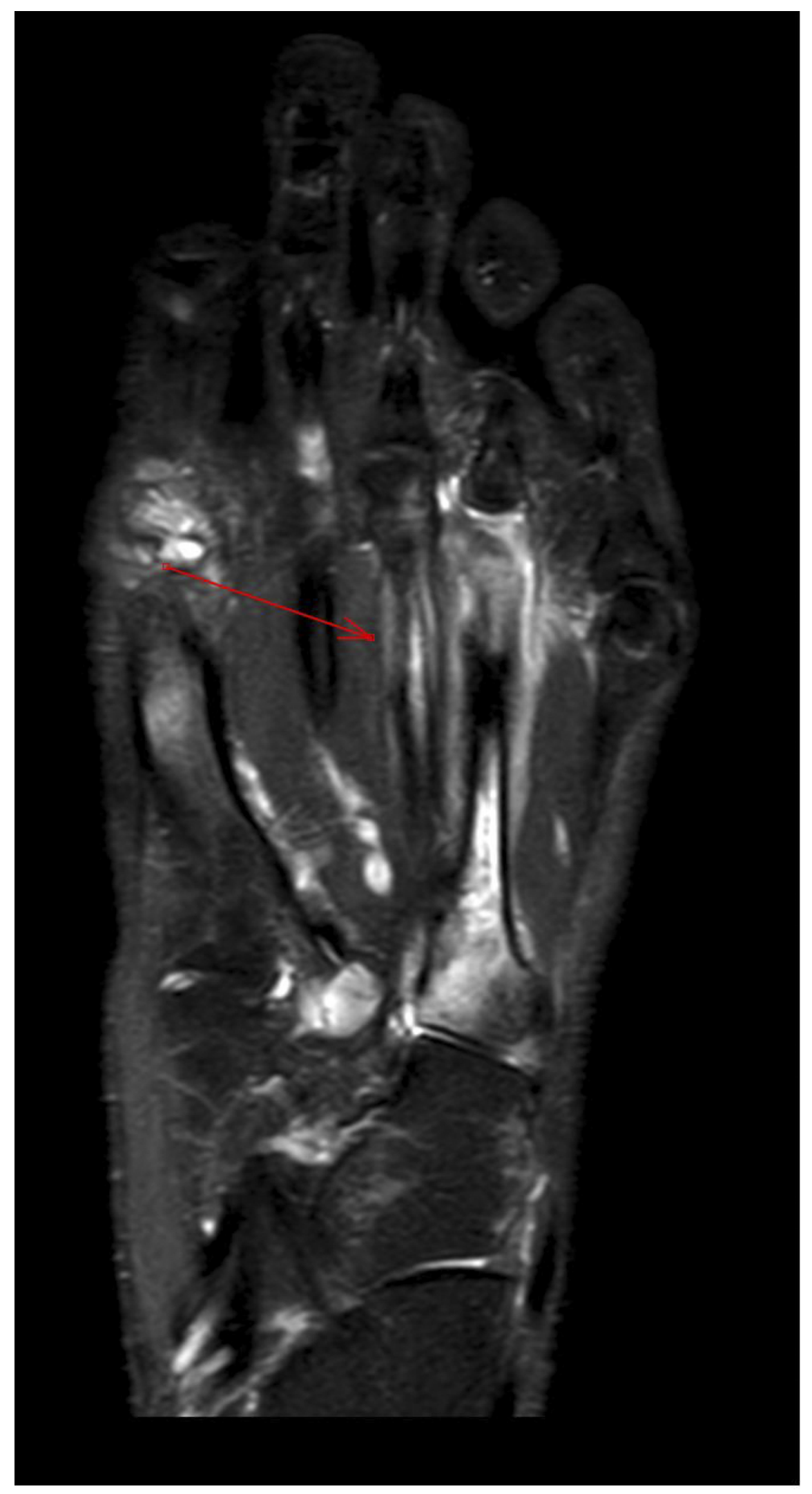

